\title{
CONTRIBUIÇÃO DOS PROGRAMAS DE COMPRA INSTITUCIONAL (PAA E PNAE) PARA A ESTABILIZAÇÃO DOS PREÇOS AGRÍCOLAS EM MINAS GERAIS
}

\author{
Contribution of institutional buying programs (PAA and PNAE) to stabilize \\ agricultural prices in Minas Gerais
}

Rafael Júnior dos Santos Figueiredo Salgado*

Erly Cardoso Teixeira*

Wellington Alvim Cunha"

Resumo: Este trabalho teve como objetivo verificar a contribuição do Programa de Aquisição de Alimentos (PAA) e do Programa Nacional de Alimentação Escolar (PNAE) para a estabilização de preços e renda dos agricultores nos mercados de arroz, feijão e milho no estado de Minas Gerais. Para tanto, foi calculado o coeficiente de variação (CV) dos preços, da renda e da quantidade produzida, considerando o período anterior e posterior à implementação dos programas. É feita também a decomposição da variância da receita, objetivando identificar qual a fonte mais importante de instabilidade da renda agrícola. Por último, foi examinada a influência dos fatores de mercado e das políticas públicas de compra institucional na determinação do preço dos produtos agrícolas. Os resultados indicaram aumento da instabilidade da receita para todos os produtos analisados, após a implementação do PAA, em decorrência, sobretudo, do aumento da variabilidade de preços. Apesar de diversos estudos apontarem estas políticas como estabilizadoras do mercado agrícola e constatar-se que foram importantes para minimizar os impactos negativos sobre a receita do produtor, o estudo constatou que ainda não apresentam-se como programas efetivos mediante a capacidade de contribuição na estabilização e elevação dos preços.

Palavras-chave: Programa de Aquisição de Alimentos, Programa Nacional de Alimentação Escolar, Estabilidade, Preços.

Abstract: We have developed this study to evaluate the contribution of the Food Acquisition Program (PAA) and the National Program for School Feeding (PNAE) to stabilize prices and incomes of farmers in the rice, beans and corn markets in Minas Gerais state. Therefore, it was calculated the coefficient of variation $(\mathrm{CV})$ to prices, income and quantity produced. In addition, it was considered the period before and after the implementation of the programs. We also have made the decomposition of the variance of income in order to identify what is the most important source of agricultural income instability. Finally, we have examined the influence of market factors and institutional purchase public policies to fix prices to agricultural products. Our results clearly indicate growth of revenue instability for all products analyzed immediately after the implementation of the PAA. This effect is due mainly to the increase in price

\footnotetext{
- Mestrando em Administração e Bacharel em Agronegócio pela Universidade Federal de Viçosa.

- Professor do Programa de Pós-Graduação em Economia Aplicada da Universidade

Federal de Viçosa. Doutor em Agricultural Economics pela Purdue Universit.

• Mestre em Administração pela Universidade Federal de Viçosa.
} 
variability. Although several studies suggest these policies as stabilizing the agricultural market and they have been described as important to minimize the negative impact on producer revenues, this study pointed them out as programs that are still not effective to contribute in the stabilization and rising prices.

Keywords: Food Acquisition Program, National School Feeding Programme, stability, prices.

JEL: Q18

\section{INTRODUÇÃO}

A agricultura familiar vem sistematicamente ganhando espaço nos debates sobre a construção coletiva de políticas públicas no Brasil, que mostram a importância desse segmento para um desenvolvimento socioeconômico mais equilibrado e sustentável no meio rural. Segundo Müller, Silva e Schneider (2012) a elaboração de políticas públicas que têm como foco a agricultura familiar contribuem para a legitimação das ações do Estado neste segmento e o reconhecimento social da importância da agricultura familiar na sociedade brasileira.

As primeiras ações nacionais voltadas para a agricultura familiar ocorreram no inicio dos anos 90 por intermédio da pressão de movimentos sociais ligados aos sindicatos rurais, que culminou no desenvolvimento do Programa Nacional de Fortalecimento da Agricultura Familiar (Pronaf) (MATTEI, 2007a). A partir deste contexto, a agricultura familiar é reconhecida como importante categoria social e política pelo Estado brasileiro (GRISA; SCHNEIDER, 2015) e passa a ser vista como segmento estratégico para a produção de matérias primas, geração de emprego e renda e abastecimento alimentar das populações.

Embora o Pronaf tenha como público alvo a agricultura familiar e abrigar um conjunto amplo de ações, envolvendo ainda comercialização, assistência técnica, extensão rural e seguro agrícola, ele se destina prioritariamente às atividades de custeio e/ou investimento e pouco beneficia a comercialização dos produtos agrícolas (NUNES, 2007). Desta forma, complementarmente, houve a necessidade da criação de um programa que priorizasse a comercialização dos produtos oriundos desse segmento. Neste contexto, em 2003, por meio da Lei Federal n. 10.696, foi criado o Programa de Aquisição de Alimentos (PAA), uma das ações estruturantes do programa Fome Zero do governo federal (YAZBEK, 2004).

O PAA foi o primeiro programa de aquisição institucional de alimentos da agricultura familiar criado pelo Brasil, possuindo três objetivos principais: (i) apoiar os agricultores familiares por meio do incentivo a produção e garantia de acesso ao mercado; (ii) distribuir alimentos para pessoas em insegurança alimentar e nutricional; e (iii) construir estoques estratégicos de produtos agrícolas. É um elemento chave na estratégia nacional de segurança alimentar e nutricional do Fome Zero, um programa multidimensional que combina o objetivo de promover a segurança alimentar com as preocupações mais amplas de desenvolvimento econômico e inclusão social (SWENSSON, 2015).

O Programa de Aquisição de Alimentos foi concebido para apoiar pequenos agricultores em um dos aspectos mais difíceis do processo produtivo: garantir acesso ao mercado para os produtos que eles produzem. $\mathrm{O}$ 
programa permite que os agricultores vendam seus produtos a instituições públicas federais, estaduais e municipais conectando grandes fontes previsíveis de demanda institucional de produtos agrícolas aos agricultores familiares, reduzindo riscos, incentivando a melhoria na qualidade do produto, aumentando a renda e reduzindo a pobreza no meio rural. Ademais, o PAA também atua na aquisição de produtos para armazenamento, regulação dos preços de mercado e assistência a grupos de indivíduos em insegurança alimentar e nutricional (INTERNATIONAL POLICY CENTER - IPC-IG, 2013; MILHORANCE; GABAS, 2015).

O PAA demonstrou a viabilidade da criação de uma demanda impulsionada pelo Estado voltada para os pequenos agricultores. Em 10 anos, ele comprou mais de 3 milhões de tonelada de alimentos de mais de 200 mil agricultores familiares. Seu orçamento - R \$ 143 milhões em 2003 - cresceu quase 10 vezes e está projetado para atingir R $\$ 1,3$ bilhões em 2015 (IPC-IG, 2013).

Já o PNAE é outra fonte de demanda institucional das organizações da agricultura familiar. Embora exista desde a década de 1950, foi apenas em 2009 que o governo federal brasileiro aprovou uma lei inovadora que redefiniu as metas da alimentação escolar, ligando-a diretamente ao desenvolvimento rural (SONNINO; TORRES; SCHNEIDER, 2014). No programa, estados, municípios e escolas federais devem investir pelo menos $30 \%$ dos recursos financeiros repassados pelo governo federal (Fundo Nacional de Desenvolvimento Escolar - FNDE) na compra direta dos produtos da agricultura familiar, preferencialmente no âmbito local, seguindo um procedimento semelhante ao adotado pelo PAA e, como tal, evitando um procedimento de licitação pública (SWENSSON, 2015; SACCO DOS ANJOS; BECKER, 2014). O PNAE investe exclusivamente na merenda escolar, como meio de promover a segurança alimentar, manter as crianças e adolescentes matriculados e melhorar o desempenho escolar, e para fortalecer a agricultura dos pequenos agricultores (IPC-IG, 2013).

O programa alimenta cerca de 43 milhões de estudantes todos os dias nas escolas públicas brasileiras e teve um orçamento anual em 2014 de aproximadamente $\mathrm{R} \$ 3,5$ bilhões. Com a Lei $\mathrm{n}^{\circ} 11.947$, de 16/06/2009, 30\% desse valor, ou seja, $\mathrm{R} \$ 1,05$ bilhão deve ser investido na compra direta de produtos da agricultura familiar, medida que estimula o desenvolvimento econômico e sustentável das comunidades, podendo, significativamente, aumentar os rendimentos agrícolas familiares e expandir as oportunidades de mercado (MDA, 2015; MEC, 2015; IPC-IG, 2013).

Considerando a importância de políticas públicas para a agricultura, diversos estudos (IPC-IG, 2013; MILHORANCE; GARBAS, 2015; DIAS; ROCHA, 2015; CERQUEIRA; ROCHA; COELHO, 2006; PEREIRA; LOURENZANI, 2014; SCHMITT, 2005; ORTEGA; JESUS; SÓ, 2007; MARTINS; CAVALCANTI, 2007) têm indicado que os programas de compra institucional têm contribuído para aumentar, regular e estabilizar os preços do mercado agrícola. Entretanto, apesar da relevância do PAA e PNAE, os estudos (CRUZ; ALVARENGA, 2015; CRUZ; TEIXEIRA, 2006; CONCEIÇÃO, 2002; CONCEIÇÃO, 2006) que analisam as contribuições de políticas governamentais para a estabilização dos preços agrícolas tem se restringindo às analises do COV, PEP e Política dos Preços Mínimos. Políticas que não alcançam os agricultores mais pobres e que afetam a uma agricultura que em 
boa medida sempre este ligada à dinâmica dos mercados regionais, nacionais e internacionais, como é o caso de commodities como a soja ou o trigo (SACCO DOS ANJOS; BECKER, 2014; GRAZIANO DA SILVA; DEL GROSSI; DE FRANÇA, 2012).

Desta forma, este trabalho vem preencher a lacuna da falta e incipiência de estudos que analisem a contribuição das novas políticas e programas públicos voltados à agricultura familiar, e sua influência sobre a estabilidade do mercado agrícola. Assim, objetivou-se verificar a contribuição do PAA e PNAE para a estabilização de preços e renda do produtor rural no estado de Minas Gerais. Paralelamente, pretendeu-se examinar a influência da oferta e demanda de mercado e variáveis da política governamental na determinação do preço.

Nesta análise, foram utilizados os produtos feijão, arroz e milho no período de 1995 a 2012. A escolha destes produtos justifica-se pelo fato desses terem sido alguns dos produtos mais comercializados em termos de valor de aquisição pelo PAA no Brasil (COMPANHIA NACIONAL DE ABASTECIMENTO - CONAB, 2012). Além de serem produtos de consumo da cesta básica, essenciais na alimentação humana e de grande valor nutricional cujo abastecimento tem sido objeto de permanente preocupação das autoridades governamentais do estado de Minas Gerais.

Optou-se por analisar o PAA em Minas Gerais, pois, dentre os estados brasileiros que acessam o programa, o estado destaca-se como uma das Unidades da Federação que recebem maior volume de recurso do programa. Em 2009, esse volume totalizou 120 milhões de reais ou $20,30 \%$ do total aplicado no país. Deve-se destacar também a predominância de duas modalidades: PAA-Leite, operacionalizada pelos Estados e Municípios do Nordeste e Norte de Minas Gerais e PAA-Compra com Doação Simultânea, operacionalizada pela Companhia Nacional de Abastecimento (Conab), sendo estas, as modalidades que receberam maior volume de recursos em Minas Gerais.

O estado também se destaca como destino do segundo maior volume de recursos no âmbito do PNAE. Em 2011, dos recursos repassados ao estado, no mínimo, $\mathrm{R} \$ 86,7$ milhões foram gastos na compra de produtos oriundos da agricultura familiar, o que pode ter impacto significativo na quantidade ofertada e demandada do mercado, influenciando os valores dos preços recebidos pelos produtores. Conjuntamente, o estado possui expressiva presença da agricultura familiar, tendo papel importante na geração de renda e emprego. Segundo o Censo Agropecuário de 2006 (INSTITUTO BRASILEIRO DE GEOGRAFIA E ESTATÍSTICA - IBGE, 2015), Minas Gerais possuía 437.415 estabelecimentos da agricultura familiar, representando $79 \%$ do total de estabelecimentos agropecuários, ocupando mais de 1,1 milhões de trabalhadores.

Apesar de sua importância, Buainain, Sabatto e Guanziroli (2004) afirmam que o universo da agricultura familiar é extremamente diferenciado e enfrenta problemas associados à disponibilidade de capital de giro e recursos para investimento. Doretto e Michelon (2007) avaliando os impactos econômicos, sociais e culturais do PAA no estado do Paraná, verificaram que os agricultores afirmam que a garantia de mercado e de preços para os produtos agropecuários foram de fundamental importância para a manutenção da renda nas unidades produtivas. Dessa forma, políticas como o PAA 
precisam ser melhor estudadas e analisadas para permitir que seus objetivos, como a inclusão da agricultura familiar ao mercado, a promoção de geração de renda, a estabilização dos preços e a dinamização da economia local/regional, sejam alcançados.

Magalhães e Soares (2006) e Maciel (2008) ressaltam que a partir do momento em que o PAA é implantado nos estados, os preços dos produtos comercializados no mercado convencional tendem a aumentar em função da retirada de uma parcela da oferta de alimentos oriundos da agricultura familiar e da manutenção da demanda. Isso se deve, pois, os beneficiários das instituições atendidas não faziam parte desse mercado. Com isso há uma elevação das receitas líquidas dos produtores. $O$ aumento na receita tende num momento seguinte, a induzir novos investimentos no setor, levando a uma aceleração do crescimento da oferta e por consequência, à retomada da tendência histórica de queda dos preços.

A Figura 1 demonstra, que ao implementar o PAA no estado de Minas Gerais, procura-se estabelecer preços nominais acima daqueles vigentes no mercado mantendo, portanto, esses preços nominais por largos períodos de tempo. Ao longo desse tempo, a inflação corrói esses valores e os preços do mercado tendem a convergir e superar em termos reais, o preço referencial fixado pelo programa.

O preço é determinante da renda, impactando assim, diretamente na capacidade de investimento e na aderência a novas tecnologias por parte dos agricultores, dessa forma, um conhecimento mais detalhado sobre os preços praticados nestes mercados pode facilitar a criação e adequação de politicas e programas públicos.

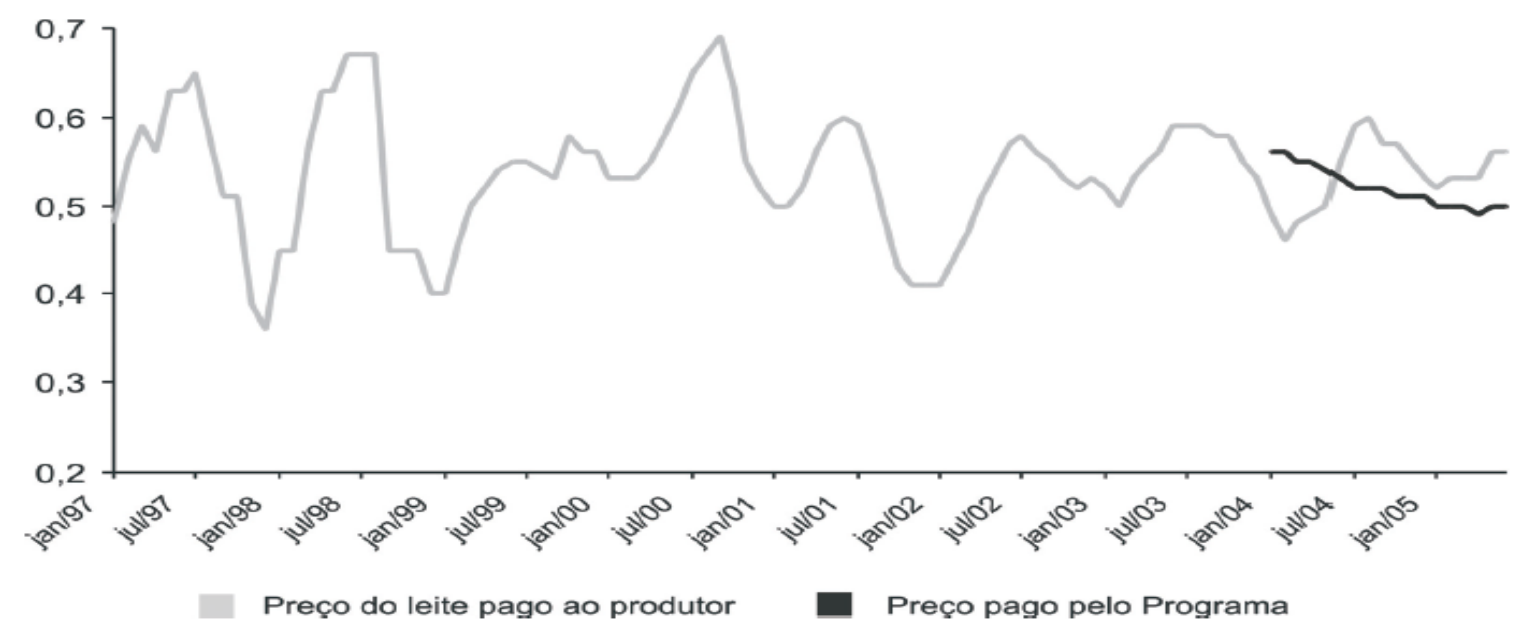

Figura 1 - Série histórica dos preços $(R \$ / L)$ do leite pago ao produtor e preços de referência do PAA.

Fonte: Magalhães e Soares (2006, p. 24).

Outro problema enfrentado pelos agricultores e que têm demandado a atenção do estado é a variabilidade dos preços, que dificulta o planejamento da produção e o abastecimento do mercado, acarretando acentuados impactos alocativos e distributivos no setor e na economia (CRUZ; TEIXEIRA, 2006). A flutuação dos preços gera aumento significativo nos riscos da atividade, reduzindo os investimentos. A variação de preços também pode causar o 
processo de transferência de renda de produtores rurais para outros segmentos presentes no fluxo de comercialização.

É nesse contexto que se destaca a importância do governo, atuando via políticas e programas públicos que busquem a estabilização dos preços, receita e ofertas agrícolas. Diante da relevância da política de compra institucional, torna-se importante fazer uma análise de seu desempenho quanto ao seu objetivo de estabilizar os preços agrícolas e consequentemente, garantir receita ao agricultor.

Após analisar o papel das políticas governamentais de estabilização dos preços agrícolas, torna-se particularmente importante obter informações sobre o comportamento desses preços e identificar as variáveis que tenham influência sobre eles.

Portanto o objetivo deste estudo, centrado em avaliar a eficácia do Programa de Aquisição de Alimentos para a estabilização dos preços do feijão, arroz e milho, principais produtos comercializados no âmbito do programa, pode ser atingido analisando-se os coeficientes de variação da receita e da decomposição de sua variância, identificando-se assim, a fonte mais importante na instabilidade da renda agrícola.

\section{METODOLOGIA}

\subsection{Variabilidade de preços agrícolas}

Segundo Conceição (2002), a variabilidade dos preços agrícolas é uma questão importante quando se pretende avaliar a eficácia dos instrumentos de comercialização. Logo, é uma importante ferramenta para verificar se o PAA contribui ou não, para a redução da volatilidade dos preços.

No presente estudo foram usados indicadores de instabilidade dos preços agrícolas, especificamente: o coeficiente de variação simples e a decomposição da variância. Segundo Cruz e Teixeira (2007), o coeficiente de variação simples é uma medida de dispersão útil para comparar variáveis cujas distribuições são diferentes e assim, atendem ao objetivo deste estudo que foi analisar as distribuições de preços e quantidades, que são distintas entre si.

Embora o desvio-padrão seja também uma medida de dispersão relativa à média, não é viável o seu uso considerando-se que as duas distribuições podem ter médias diferentes, o que torna os desvios dessas duas distribuições não comparáveis. Por isso, a solução será usar o coeficiente de variação, que é igual ao desvio-padrão $(\sigma)$ dividido pela média $(\mu)$, conforme expressão 1 :

$$
\text { Coeficiente de variação simples }(\mathrm{CV})=\frac{\sigma(x)}{\mu(x)}
$$

Para Pimentel-Gomes (2009), os valores dos coeficientes de variação simples são considerados baixos se forem inferiores a 10\%; médios, quando situados entre $10 \%$ e $20 \%$; altos, quando estiverem entre $20 \%$ e $30 \%$ e muito altos, quando superiores a $30 \%$.

O segundo método analítico utilizado foi a decomposição da variância. Neste estudo foi utilizada para explicar a variabilidade da receita (proxy de renda) dos produtores rurais. Para Cruz e Teixeira (2007), este método permite apontar qual componente da receita agrícola (preço ou quantidade) possui 
maior influência sobre a instabilidade da receita total do setor agrícola, em diferentes períodos do tempo. A importância relativa da variância do preço e da quantidade na variância total da receita permite identificar a contribuição de cada componente para a variabilidade da receita do setor agrícola. Considerando que a receita total pode variar devido às alterações nos preços, mantidas as quantidades constantes (média), ou devido às alterações nas quantidades, mantidos os preços constantes (média), pode-se definir a variância da receita total desta forma:

$$
\begin{aligned}
\sigma_{r}^{2} & =\operatorname{VAR}\left(P^{*} Q\right) \\
\sigma^{2}{ }_{r} & =\operatorname{VAR}\left(P^{*} Q\right)=\operatorname{VAR}(\bar{P} Q+\bar{Q} P)=\mathrm{E}[\bar{P} Q+\bar{Q} P-\mathrm{E}(\bar{P} Q+\bar{Q} P)]^{2} \\
\sigma_{r}{ }_{r} & =\operatorname{VAR}(\bar{P} Q+\bar{Q} P)=\bar{P}^{2} \operatorname{var}(Q)+\bar{Q}^{2} \operatorname{var}(P)+2 \bar{P} Q \operatorname{cov}(Q, P) \\
\sigma_{r}{ }_{r} & =V A R(\bar{P} Q+\bar{Q} P)=\bar{P}^{2} \operatorname{var}(Q)+\bar{Q}^{2} \operatorname{var}(P)+2 \bar{P} \bar{Q} \rho \sigma_{P} \sigma_{Q}
\end{aligned}
$$

em que $\bar{P}$ é o preço médio do produto; $\bar{Q}$, a quantidade média produzida; $\rho$, o coeficiente de correlação entre preço e quantidade; $\sigma_{P}$, o desvio padrão do preço; e ${ }^{\sigma_{q}}$, o desvio padrão da quantidade.

O período de análise é de 1995 a 2012. Para atender aos objetivos propostos no trabalho este período foi segmentado e para cada subperíodo foram calculados os indicadores citados anteriormente. Para estimar o impacto do Programa de Aquisição de Alimentos sobre a variabilidade dos preços, o período foi dividido em dois subperíodos, antes do Programa 1995/2002 e durante o Programa 2003/2012. No período de 1995 a 2002, a economia nacional sofreu extremas e importantes transformações tais como, a abertura econômica e crescente inserção da economia brasileira no comércio mundial, além da implementação do Plano Real e da criação do Prêmio para Escoamento de Produto (PEP) e dos Contratos de Opção de Venda (COV). No subperíodo 2003-2012, políticas de comercialização dos produtos da agricultura familiar foram implementadas, visando garantir mercado e renda aos agricultores familiares e estabilizar os preços agrícolas nos mercados locais. Todos estes fatores causaram impactos significativos sobre a instabilidade da receita agrícola.

Nos subperíodos 2009/2011 ocorre a promulgação da Lei $n^{\circ} 11.947$, de 16 de junho de 2009 que em seu Artigo 14 determina que do total dos recursos financeiros repassados pelo Fundo Nacional de Desenvolvimento da Educação (FNDE), no âmbito do Programa Nacional de Alimentação Escolar (PNAE), no mínimo $30 \%$ deverão ser utilizados na aquisição de gêneros alimentícios diretamente da agricultura familiar. Teoricamente, nesse período há reflexos sobre a volatilidade dos preços agrícolas. Portanto o período 2009/2012 procurou medir o impacto dessa nova política de comercialização sobre a variabilidade dos preços agrícolas recebidos pelos agricultores familiares.

\subsection{Modelo de Preços}


O modelo estatístico utilizado no estudo refere-se ao Modelo de Regressão Linear Múltiplo, visto que este é explicado por mais de uma variável independente. Conforme Santana (2003), este modelo consiste em uma técnica econométrica que pode ser utilizada para analisar a relação entre uma variável dependente e duas ou mais variáveis independentes.

O modelo empírico utilizado baseia-se no trabalho de Conceição (2006), no qual a autora buscou verificar a influência de instrumentos governamentais na determinação de preços dos produtos agrícolas. Objetiva-se estimar quais variáveis têm maior influência sobre a determinação dos preços recebidos pelo produtor rural, onde foi analisado as influências dos fatores oferta de mercado, preço de mercado defasado e variáveis de políticas públicas governamentais na determinação dos preços do arroz, feijão e milho.

O modelo empírico a ser utilizado na estimação será o seguinte:

Modelo para Arroz:

$L N P a r r=\alpha_{0}+\alpha_{1}$ Ln Qarr $+\alpha_{2}$ LnPDEFarr $+\alpha_{3} \operatorname{Ln} D_{1}$ PPAAarr $+\mu$

Modelo para Feijão:

$L N P f e i j=\alpha_{0}+\alpha_{1} \operatorname{Ln} Q f e i j+\alpha_{2} L n P D E F f e i j+\alpha_{3} L n D_{1} P P A A f e i j+\mu$

Modelo para Milho:

LNPmil $=\alpha_{0}+\alpha_{1}$ Ln Qmil $+\alpha_{2}$ LnPDEFmil $+\alpha_{3} \operatorname{Ln} D_{1} P P A A m i l+\mu$

em que:

Parr = preço do arroz recebido pelo produtor;

Qarr = quantidade produzida de arroz;

PDEFarr = preço defasado do arroz;

PPAAarr = preço PAA do arroz;

Pfeij = preço do feijão recebido pelo produtor;

Qfeij = quantidade produzida de feijão;

PDEFfej = preço defasado do feijão;

PPAAfeij = preço PAA do feijão;

Pmil = preço do milho recebido pelo produtor;

Qmil = quantidade produzida de milho;

PDEFmil = preço defasado do milho;

PPAAmil = preço PAA do milho.

$\mathrm{D}_{1}=$ variável dummy igual a zero no período 1995-2002 período sem 0 PAA e 1 no período 2003-2012 período com o PAA.

A variável $Q$ é comumente utilizada em modelos de preços e fornece medida da influência das forças de mercado na determinação dos preços. $O$ sinal esperado para esta variável é negativo. Isso porque, se a oferta aumentar, os preços tendem a cair. Por outro lado, se a oferta diminuir, os preços de mercado tendem a aumentar.

A interação da variável preço do PAA com a variável dummy será feita para tentar identificar o impacto do programa. A variável dummy foi definida como igual a 0 no período sem o PAA, 1995-2002, e 1 no período com o PAA 2003-2012. Os modelos são estimados por Mínimos Quadrados Ordinários (MQO).

Os dados dos preços recebidos pelos produtores de arroz, feijão e milho foram obtidos junto à Fundação Getúlio Vargas (FGV/DADOS), para o período de 1995 a 2012. Considerada a possibilidade de existência de informações incorretas e a interpretação do comportamento das informações, os dados de 
preço foram deflacionados utilizando-se o Índice Geral de PreçosDisponibilidade Interna (IGP-DI) da Fundação Getúlio Vargas. Quanto aos dados referentes às quantidades produzidas, foram coletados junto à Companhia Nacional de Abastecimento. Escolheu-se este período de análise tendo em vista o objetivo de se verificar o impacto dos novos instrumentos de comercialização sobre a variabilidade dos preços, quantidades produzidas e receita.

\section{RESULTADOS E DISCUSSÃO}

\subsection{Variabilidade de preços agrícolas: Coeficientes de Variação}

Nesta seção, calcula-se o coeficiente de variação (CV) para as quantidades produzidas, para as receitas e preços médios auferidos pelos produtores de arroz, feijão e milho no período 1995-2012. São feitas análises para os seguintes subperíodos: 1995-2002, 2003-2012, 2003-2008 e 20092012.

A análise dos subperíodos 1995-2002 e 2003-2012 tem por objetivo comparar a variabilidade da receita agrícola diante da implantação de políticas públicas de extrema importância para a economia nacional e principalmente para a agricultura familiar. No período de 1995-2002, que abrange período pósPlano Real, os preços agrícolas sofreram fortes quedas, decorrente da estabilização da política econômica adotada e do aumento das importações de commodities diante do câmbio sobrevalorizado e do cenário de grande liquidez externa, incorrendo em perdas aos agricultores em virtude da diminuição dos preços e consequentemente da receita agrícola (CRUZ; TEIXEIRA, 2006). Além disso, esse período é marcado pela criação do Pronaf em 1995, primeira política nacional de fortalecimento da agricultura familiar (MATTEI, 2007b), entretanto este programa não abordava a comercialização dos produtos da agricultura familiar, historicamente, o calcanhar de Aquiles no desenvolvimento da atividade.

Andrews (2004) ressalta que o sistema de crédito advindo com o Pronaf enfrentou dificuldades de implementação junto aos agricultores mais vulneráveis economicamente. Em virtudes dessas questões, aliadas à transição de governos na esfera federal no ano 2001/2002, houve a necessidade de criação e fortalecimento de novas intervenções que fossem capazes de ampliar os benefícios socioeconômicos aos produtos rurais de menor renda e que auxiliasse a comercialização dos produtos oriundos da agricultura familiar (SIMÃO; SILVA; SILVEIRA, 2014). Em decorrência analisese o subperíodo 2003-2012, período de criação e implementação do Programa de Aquisição de Alimentos, garantindo benefícios aos agricultores como apoio à produção para autoconsumo, do excedente para comercialização e do subsídio ao consumo, beneficiando diretamente os produtores através da recuperação dos preços de mercado dos produtos agrícolas.

Já a análise dos subperíodos 2003-2008 e 2009-2012 tem por objetivo avaliar a variabilidade da receita agrícola no estado de Minas Gerais, diante da implementação do Programa Nacional de Alimentação Escolar (PNAE) e consequente incentivo à produção de produtos agrícolas na agricultura familiar para atender à cota mínima de fornecimento de $30 \%$ de produtos agrícolas oriundos da agricultura familiar para a alimentação escolar, visto que, esta 
política pode ter causado impactos significativos sobre a instabilidade da receita agrícola.

No período 2003-2008, não havia mercado institucional de alimentos para a merenda escolar, ou seja, a demanda institucional de produtos da agricultura familiar era oriunda exclusivamente do Programa de Aquisição de Alimentos, que tinha influência relativamente menor na quantidade produzida e no preço recebido pelos produtores.

A partir de 2009, a Lei $n^{\circ} 11.947 / 2009$ entra em vigor e determina a utilização de no mínimo, 30\% dos recursos repassados pelo Fundo Nacional de Desenvolvimento da Educação (FNDE) para alimentação escolar, aplicados na compra de produtos da agricultura familiar e do empreendedor rural dispensando o procedimento licitatório.

No ano de 2011 , foram repassados $R \$ 3,118$ bilhões de reais às prefeituras e desse total, no mínimo $\mathrm{R} \$ 936,304$ milhões deveriam ser empenhados na compra de alimentos da agricultura familiar. Em Minas Gerais, $\mathrm{R} \$ 86,785$ milhões no mínimo, foram gastos na compra de produtos oriundos da agricultura familiar, o que pode ter impacto significativo na quantidade ofertada e demanda do mercado, impactando nos valores dos preços recebidos pelos produtores.

Diante da implantação destas políticas, visando distinguir os efeitos delas na receita agrícola, são feitas análises dos subperíodos definidos anteriormente. Os coeficientes de variação para as receitas agrícolas e de seus componentes para todos os subperíodos estão apresentados na Tabela 1.

Tabela 1 - Coeficientes de variação de preços recebidos, quantidade e receita dos produtores de arroz, feijão e milho em Minas Gerais.

\begin{tabular}{cccccccccc}
\hline \multirow{2}{*}{ Período } & \multicolumn{3}{c}{ Arroz } & \multicolumn{3}{c}{ Feijão } & \multicolumn{4}{c}{ Milho } \\
\cline { 2 - 10 } & Preço & Produção & Receita & Preço & Produção & Receita & Preço & Produção & Receita \\
\hline $1995-2002$ & $13 \%$ & $41 \%$ & $40 \%$ & $26 \%$ & $9 \%$ & $19 \%$ & $12 \%$ & $7 \%$ & $11 \%$ \\
$2003-2012$ & $22 \%$ & $39 \%$ & $47 \%$ & $28 \%$ & $11 \%$ & $33 \%$ & $17 \%$ & $10 \%$ & $17 \%$ \\
$2003-2008$ & $23 \%$ & $19 \%$ & $25 \%$ & $27 \%$ & $8 \%$ & $33 \%$ & $13 \%$ & $9 \%$ & $16 \%$ \\
$2009-2012$ & $14 \%$ & $30 \%$ & $43 \%$ & $32 \%$ & $6 \%$ & $38 \%$ & $15 \%$ & $9 \%$ & $20 \%$ \\
\hline
\end{tabular}

Fonte: Elaboração própria.

Analisando-se os dados de coeficiente de variação, nota-se que no período de 2003 a 2012, houve aumento dos coeficientes de variação da receita agrícola do arroz, feijão milho. No mercado de milho, o aumento da variabilidade da receita dos produtores está ligada ao aumento na variabilidade do preço e da produção. Já o aumento da variabilidade da receita dos produtores de arroz foi causado pelo aumento significativo do CV dos preços, uma vez que, o coeficiente de variação da produção não aumentou significativamente entre 1995 e 2003.

No mercado de feijão, há um aumento da variabilidade da receita dos produtores no período 2003-2012 em relação a 1995-2002. Houve aumento pouco significativo na instabilidade do preço recebido pelo produtor neste mercado e uma pequena variação na instabilidade da quantidade produzida.

De acordo com os intervalos de valores de coeficientes de variação propostos por Pimentel-Gomes (2009), pode-se afirmar que, entre 2003-2012, os coeficientes referentes às receitas dos produtores de arroz e do feijão 
mostram-se muito elevados, com níveis acima de $30 \%$, devido, principalmente, ao elevado CV do preço no caso do feijão e ao alto CV da produção no caso do arroz. No mercado de milho o CV indicam nível médio de variabilidade.

Na sequência, são feitos recortes temporais, com o objetivo de isolar os efeitos do PNAE, que pode ter influenciado a demanda e oferta dos produtos analisados e, consequentemente, a estabilidade da receita agrícola. Para tal, é feita a comparação da estabilidade identificada entre 2003-2008 em relação aquela presente em 2009-2012. Enfatiza-se que, a partir de 2009 entra em vigor a Lei $\mathrm{n}^{\circ} 11.947$ determinando que $30 \%$ dos recursos do FNDE repassados aos municípios para compra da merenda escolar, sejam utilizados na compra de produtos oriundos da agricultura familiar.

De acordo com a Figura 2, observa-se que no mercado de arroz, os produtores se defrontam com menor estabilidade de preços após as mudanças no PNAE. No entanto, a variação da produção aumentou o que contribuiu para aumento na variabilidade da receita dos produtores no período 2009-2012. O aumento da volatilidade da produção não é compensado pela queda na variabilidade dos preços.

No caso do setor do milho, percebe-se que não houve impacto imediato na produção. Há um aumento da instabilidade da receita em razão do aumento da variabilidade dos preços, pois o coeficiente de variação da produção de milho mantém-se praticamente idêntico ao do período anterior.

Ainda em relação à Figura 2, percebe-se que os produtores de feijão obtiveram receitas muito instáveis no período 2009-2012 em relação a 20032008. O aumento na instabilidade dos preços é elevado e contribui fortemente para o aumento na volatilidade da receita agrícola do mercado de feijão, mesmo havendo uma pequena diminuição na instabilidade da produção, que não compensa o aumento na volatilidade de preços.

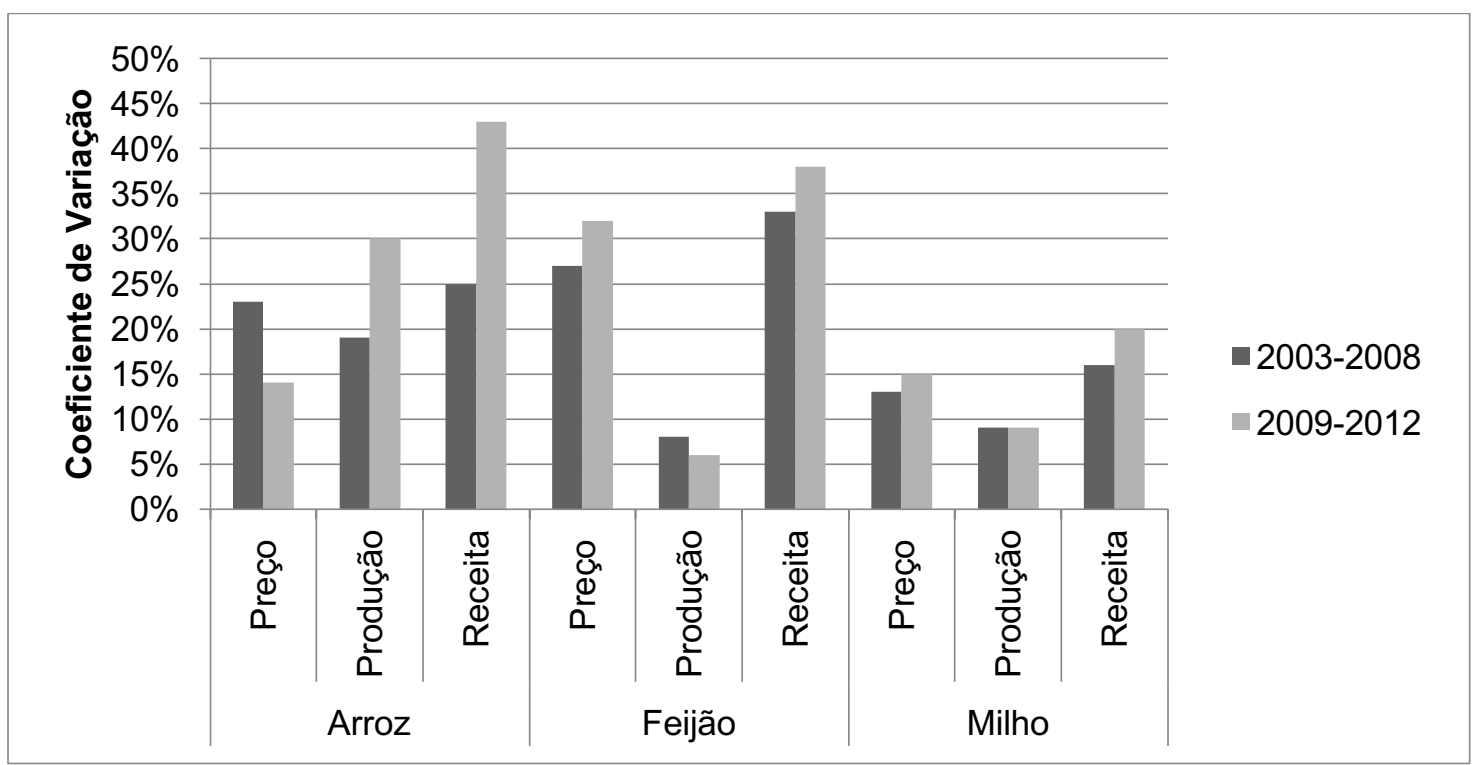

Figura 2 - Coeficientes de variação (em \%) para mercados de arroz, feijão e milho, 2003-2008 e 2009-2012.

Fonte: Elaboração própria.

Pode-se afirmar que há menor estabilidade da receita agrícola após a Lei $n^{\circ} 11.947$ entrar em vigor, esse resultado pode ter sido influenciado pelo cenário de crise mundial, que impactou fortemente a economia brasileira, 
principalmente o segmento agrícola com o aumento do endividamento dos produtores e consequentemente do custo da dívida (CRUZ; ALVARENGA, 2015), gerando instabilidade econômica e fazendo com que as mudanças no PNAE não contribuíssem efetivamente para que a eficácia da política de incentivo à comercialização dos produtos da agricultura familiar e estabilização dos preços agrícolas.

Seguindo o critério de Pimentel-Gomes (2009) sobre intervalos definidos para valores dos CV, observa-se que, no período 2009-2012, os coeficientes de variação da produção enquadram-se como baixos nos mercados de feijão e milho, pois são inferiores a $10 \%$ e alto no mercado de arroz. Os CV referentes a preços podem ser considerados médio nos mercados de milho e arroz, pois estão situados entre $10 \%$ e $20 \%$ e muito alto no mercado de feijão. Os mercados de arroz e feijão possuem CV da receita agrícolas muito altos, indicando alta instabilidade da receita nesses setores, somente o mercado de milho apresenta CV médio (20\%). Denota-se que o milho, que é contemplado em políticas especifica de redução da instabilidade tais como o COV e PEP, têm sofrido menor oscilação na variabilidade de preços e consequentemente da receita.

\subsection{Decomposição da variância}

Nesta seção é feita a decomposição da variância da receita obtida nos mercados agrícolas de arroz, feijão e milho. Conforme informado, este método permite desagregar a variância da receita recebida pelos produtores em seus componentes: preço e quantidade produzida, além de considerar a interação entre tais componentes. O termo de interação representa o efeito resultante da variação simultânea dos componentes preços e quantidade, captando a correlação que existe entre os mesmos. No que se refere aos intervalos temporais, permanece a subdivisão feita inicialmente. Os resultados relativos à participação relativa de cada componente na variabilidade da receita total estão contidos na Tabela 2.

Tabela 2 - Decomposição da variância da receita dos produtores de arroz, feijão e milho (\%), Minas Gerais, por subperíodos.

\begin{tabular}{|c|c|c|c|}
\hline \multirow{2}{*}{ Produto } & Variação do Preço & Variação da Produção & Interação \\
\hline & \multicolumn{3}{|c|}{ Período 1995-2002 } \\
\hline Arroz & $11 \%$ & $103 \%$ & $-6 \%$ \\
\hline Feijão & $183 \%$ & $20 \%$ & $-76 \%$ \\
\hline \multirow[t]{2}{*}{ Milho } & $116 \%$ & $45 \%$ & $-60 \%$ \\
\hline & \multicolumn{3}{|c|}{ Período 2003-2012 } \\
\hline Arroz & $20 \%$ & $63 \%$ & $-35 \%$ \\
\hline Feijão & $67 \%$ & $10 \%$ & $15 \%$ \\
\hline \multirow[t]{2}{*}{ Milho } & $99 \%$ & $38 \%$ & $72 \%$ \\
\hline & \multicolumn{3}{|c|}{ Período 2003-2008 } \\
\hline Arroz & $87 \%$ & $55 \%$ & $-40 \%$ \\
\hline Feijão & $67 \%$ & $6 \%$ & $22 \%$ \\
\hline Milho & $69 \%$ & $33 \%$ & $4 \%$ \\
\hline
\end{tabular}




\begin{tabular}{cccc} 
Arroz & $11 \%$ & $46 \%$ & $40 \%$ \\
Feijão & $72 \%$ & $2 \%$ & $18 \%$ \\
Milho & $56 \%$ & $20 \%$ & $29 \%$ \\
\hline
\end{tabular}

Fonte: Elaboração própria.

Analisando a Tabela 2, é importante destacar que no período de 19952002, a variância de preço domina a explicação da variação da renda total dos produtores, nos mercados de feijão e milho, exceto no mercado de arroz. Este período possui maior instabilidade dos preços, quando comparado ao período de 2003-2012. No setor de arroz a variância de produção tem maior impacto que o termo de interação e variância de preços, sobre a variabilidade da receita dos produtores. Denota-se que a interação entre a variância da produção e dos preços se mostrou significativa na redução da volatilidade da receita para todos os produtos analisados neste período.

A avaliação do subperíodo 2003-2012 mostra, primeiramente, que o preço deixa de ser a variável mais importante como fonte de instabilidade da receita no mercado de arroz. Isso remete aos efeitos positivos do Programa de Aquisição de Alimentos e da ação de novos instrumentos institucionais de comercialização agrícola sobre a estabilidade dos preços agrícolas nesse setor. Neste mercado, a variável quantidade é a variável de maior expressão na instabilidade da receita dos produtores. Nos mercados de feijão e milho, a variável preço continua sendo o termo de maior expressão na instabilidade da receita dos produtores desses setores. No mercado de feijão a variável produção atinge somente $10 \%$. Observa-se também que a interação entre as variâncias de preço e de quantidade se mostrou significativa na redução da volatilidade da receita dos produtores de arroz.

No período 2003-2008, marcado pela implantação do Programa de Aquisição de Alimentos, a instabilidade do preço age com maior influência para elevar a variância de receitas nos mercados dos produtos analisados. No caso da importância relativa da variabilidade da produção no mercado de feijão, o método de decomposição da variância aponta poder explicativo baixo para este componente, dado o valor próximo aos $10 \%$. No que tange à parcela de contribuição do componente de interação entre variâncias de preço e quantidade, no mercado de arroz, infere-se como significativo para reduzir a instabilidade da receita dos produtores desta cultura, enquanto que, no mercado de feijão o componente de interação aumenta a instabilidade da receita agrícola deste setor. A interação entre variâncias da produção e dos preços não se mostrou significativa para influenciar a variância da receita do mercado de milho.

Quando analisados os resultados da decomposição da variância, tendo como base os dados do subperíodo 2009-2012 marcado pela vigência da Lei $\mathrm{n}^{\circ}$ 11.947, observa-se que o PNAE foi eficiente para estabilizar os preços agrícolas apenas do mercado de arroz, tendo em vista que a variância dos preços deixa de ser a principal fonte de instabilidade da receita agrícola neste período. Nos mercados de feijão e milho, a variância do preço é superior à variância dos demais componentes, sendo que no setor de feijão esta contribuição é elevada.

Em síntese, os resultados, a partir da decomposição da variância, permitem concluir que o PAA mostrou-se eficaz apenas no mercado de arroz, quanto à meta de reduzir a instabilidade das receitas, quantidades e preços 
agrícolas, após o período de sua implementação. De maneira positiva, a vigência da Lei $n^{\circ} 11.947$, que determina a compra da agricultura familiar de $30 \%$ recursos repassados para compra da merenda escolar aos municípios via PNAE, mostra-se relevante apenas para o mercado de arroz ajudando a diminuir a estabilidade dos preços agrícolas deste setor.

\subsection{Modelos de preços e indicadores de correlação}

Nesta seção busca-se investigar a influência de algumas variáveis sobre a determinação do preço ao produtor através da estimação de modelos para o arroz, feijão e milho. O ajustamento dos modelos estimados foi feito através do Método dos Mínimos Quadrados. O número de observações variou de acordo com cada modelo devido à dificuldade prática de encontrar todos os dados necessários (variáveis independentes) para todos os anos. Os problemas de heterocedasticidade e autocorrelação foram corrigidos pelo método de White. As Tabelas 3, 4 e 5 sintetizam os resultados encontrados. A Tabela 3 apresenta os resultados dos determinantes do preço do arroz.

Tabela 3 - Resultados do modelo para o arroz 1995-2012

\begin{tabular}{lcc}
\hline Variável & Coeficiente & Erro-padrão \\
\hline Lnqarroz & $-0,0335965^{\mathrm{ns}}$ & 0,1113778 \\
Inpaaarroz & $-0,0068599^{\mathrm{ns}}$ & 0,0169784 \\
def_arr & $0,2678613^{\mathrm{ns}}$ & 0,2396287 \\
constante & $5,35^{*}$ & 1,951376 \\
& $\mathrm{R}^{2}=0,12$ & Valor $\mathrm{F}=0,66$
\end{tabular}

Fonte: Elaboração própria.

Nota: Inqarroz - representa o LN da quantidade de arroz produzido em MG no período; Inpaaarroz - LN do preço do Programa de Aquisição de Alimentos para o arroz ; def_arr - preço de mercado do arroz defasado em um período; Significância dos coeficientes: * $10 \%,{ }^{\text {ns }}$ não significante.

No modelo estimado para o arroz, a variável que procurou verificar o impacto do Programa de Aquisição de Alimentos sobre o preço não se mostrou significativa, apresentando sinal condizente com a teoria, uma vez que, um aumento substancial nos preços praticados pelo programa implica em redução dos preços no mercado de arroz, que neste caso seria de 0,7\%. Para Magalhães e Soares (2006), o aumento na receita do produtor por meio da venda e acesso ao mercado do PAA tende num momento seguinte, a induzir novos investimentos no setor, levando a uma aceleração do crescimento da oferta e por consequência, à retomada da tendência histórica de queda dos preços. A variável produção não mostrou-se significativa e com sinal negativo, indicando que um aumento na produção de arroz impactou negativamente sobre os preços do arroz, efeito em consonância com a teoria. A não significância dos coeficientes pode ser atribuída ao pequeno período de tempo considerado e possivelmente, a qualidade dos dados.

Os resultados para o modelo do feijão podem ser visualizados na Tabela 4. O sinal da variável Inpaafeijo é negativo e esperado, mostrando que o PAA teve impacto negativo sobre os preços do feijão após sua implantação. A variável que mede o impacto da produção sobre o preço não teve sinal esperado e não foi significativo. 
Tabela 4 - Resultados do modelo para o feijão 1995-2012

\begin{tabular}{lcc}
\hline Variável & Coeficiente & Erro-padrão \\
\hline Lnqfeijo & $-0,4485389^{\mathrm{ns}}$ & 0.6772541 \\
Inpaafeijo & $-0,005095^{\mathrm{ns}}$ & 0.0203369 \\
def_feij & $-0,1442461^{\mathrm{ns}}$ & 0.2520746 \\
constante & $14,6258^{\mathrm{ns}}$ & 9.698309 \\
& $\mathrm{R}^{2}=0,13$ & Valor $\mathrm{F}=0,6294$
\end{tabular}

Fonte: Elaboração própria.

Nota: Inqfeijo - representa o LN da quantidade de feijão produzida em MG no período; Inpaafeijo - LN do preço do Programa de Aquisição de Alimentos para o feijão; def_feij - preço de mercado do feijão defasado em um período;

Significância dos coeficientes: ${ }^{*} 10 \%,{ }^{\text {ns }}$ não significante.

No modelo estimado para o milho (Tabela 5), apenas a variável Inpaamilho apresentou resultado significativo. A variável que procurou verificar o impacto da implantação do PAA se mostrou significativa, apresentando sinal condizente com a teoria. Isto é, a variável Inpaamilho mostra que um aumento de $1 \%$ nos preços do PAA de milho causa efeito negativo nos preços do milho. As variáveis Inqmilho e def_mil não se mostraram significativas.

Tabela 5 - Resultados do modelo para o milho 1995-2012

\begin{tabular}{lcc}
\hline Variável & Coeficiente & Erro-padrão \\
\hline Inqmilho & $0,2241633^{\text {ns }}$ & 0,2866392 \\
Inpaamilho & $-0,0382272^{*}$ & 0,0186498 \\
def_mil & $-0,0170048^{\text {ns }}$ & 0,2895462 \\
constante & $2,898117^{\text {ns }}$ & 5,109401 \\
& $\mathrm{R}^{2}=0,35$ & Valor $\mathrm{F}=0,13$ \\
\hline
\end{tabular}

Fonte: Elaboração própria.

Nota: Inqmilho - representa o LN da quantidade de milho produzido em MG no período; Inpaamilho - LN do preço do Programa de Aquisição de Alimentos para o milho ; def_mil - preço de mercado do milho defasado em um período;

Significância dos coeficientes: * $10 \%,{ }^{\text {ns }}$ não significante.

Os resultados encontrados através da estimação dos modelos indicaram que o PAA não tem garantido a sustentação e aumento dos preços dos produtos analisados. Este resultado corrobora com os resultados anteriormente evidenciados, mostrando que, apesar de ser uma política importante para atenuar as dificuldades do produtor, principalmente 0 processo de comercialização da produção, ele ainda não tem sido suficiente e eficiente na estabilização dos preços e da receita agrícola.

\section{CONCLUSÕES}

Com base nos resultados obtidos neste trabalho, as políticas públicas de compra institucional de alimentos foram importantes para minimizar os impactos negativos sobre a receita do produtor. Entretanto, estes programas não se mostraram suficientes para diminuir a instabilidade da receita nos mercados dos produtos analisados. Tal conclusão se fundamenta no aumento dos coeficientes de variação para o arroz, feijão e milho. 
O aumento da instabilidade agrícola destes mercados se deve sobremaneira ao aumento da instabilidade dos preços que continuou em patamar elevado no período de 2003 a 2012 em relação a 1995 a 2002. Inferese que a implementação do Programa de Aquisição de Alimentos, a partir de 2003, e do PNAE, a partir de 2009, em conjunto com as políticas públicas de estabilização dos preços, COV e PEP, não foram suficientes para permitir e contribuir com uma maior estabilidade aos preços agrícolas e consequentemente, às rendas agrícolas dos produtos analisados.

Apesar de diversos trabalhos evidenciarem o PAA como instrumento de garantia de renda e sustentação e elevação de preços, este estudo mostra que ele não tem conseguido atingir o objetivo de estabilizar os preços dos produtos agrícolas analisados. Observa-se diversos fatores críticos que limitaram o sucesso da intervenção governamental, fatores esses relacionados a não continuidade do programa, relatado no estudo de Salgado e Dias (2013), ao limitado número de agricultores participantes e ao baixo volume de recurso disponibilizado, reduzindo o potencial impacto do programa na estabilidade dos preços e renda agrícola.

O PNAE conseguiu reduzir a instabilidade de preços, porém apenas no mercado de arroz. Esta estabilidade ainda é pouco significativa, pois o preço continua em patamares elevados, combinada a um cenário em que a Política de Garantia de Preços Mínimos fica condicionada a cobrir os custos de produção. Nesse mesmo sentido, a produção vem oscilando, principalmente no mercado do feijão, causando incerteza aos agricultores, principalmente os familiares, que buscam em outras culturas ou atividades maior rentabilidade e estabilidade de mercado.

Apesar de não contribuir efetivamente para a estabilização dos mercados agrícolas, a analise dos resultados obtidos pelos modelos estimados mostrou que, dentre as variáveis utilizadas, a que teve maior influência na explicação dos preços do arroz, feijão e milho foi a implementação no PAA, principalmente entre 2003 a 2009, período que não sofreu efeitos da crise econômica mundial de 2008. No caso dos produtos analisados observou-se sinal esperado, em acordo com a teoria, indicando que o aumento substancial nos preços do PAA tem impactado negativamente no preço deste produto.

Denota-se que a criação dos mercados institucionais (PNAE e PAA) trouxe consigo um vetor importante de mudanças. Ainda que limitado pela dimensão do programa e pelo número de agricultores participantes, propiciou e segue propiciando, uma elevação geral dos preços nos espaços de comercialização. É necessário incrementar o investimento para consolidar e ampliar essas experiências, não somente em relação a quantidade de recursos para a continuidade e ampliação dos projetos existentes, mas também, tendo em vista a acumulação de experiências, para propor a realização de políticas complementares.

Como limitações desta pesquisa deve-se destacar a inexistência ou desconhecimento de estudos similares no Brasil ou para outros Estados, isso impede que se possam apoiar as argumentações aqui inseridas, fazendo contraposições ou confirmações acerca de achados que se consideram essenciais para ratificar os resultados encontrados nessa pesquisa. Também foram encontradas limitações relacionadas, principalmente, à falta de dados consolidados e atualizados sobre o Programa de Aquisição de Alimentos e o Programa Nacional de Alimentação Escolar no estado de Minas Gerais. Ainda, 
houve indisponibilidade de preços dos produtos analisados para todos os municípios mineiros no período de 2003 a 2012, uma vez que diversos municípios não tiveram acesso continuo ao programa e vários nunca $o$ acessaram.

Diante do exposto, há de ressaltar o mérito do presente estudo diante da contribuição de pesquisa com evidências ligadas à volatilidade da variabilidade dos agentes envolvidos com os mercados de arroz, feijão e milho. No entanto, há a necessidade de realização de estudos que envolvam outras variáveis de mercado e de estrutura de produção como consumo, gastos com insumo, quantidade comercializada, entre outros fatores igualmente importantes. Neste sentido, estudos que possam explicar as relações de mercado, de produção e de aumento de área relacionadas à produção agrícola devem ser elaborados e discutidos, considerando que a compra institucional de alimentos da agricultura familiar é um mercado recente e precisa de políticas adicionais sólidas e específicas voltadas ao seu dinamismo.

A implementação do PAA e PNAE aponta, portanto, para a necessidade de mais estudos que possam contribuir para sua consolidação enquanto políticas públicas eficientes e que contribuam com a estabilização da renda agrícola, principalmente aos agricultores familiares, contribuindo para 0 desenvolvimento rural no Brasil.

\section{REFERÊNCIAS}

ANDREWS, C. W. Anti-poverty policies in Brazil: reviewing the past ten years. International Review of Administrative Sciences, v. 70, n. 3, p. 477-488, 2004.

BUAINAIN, A. M.; SABATTO, A.; GUANZIROLI, C. E. Agricultura familiar: um estudo de focalização regional. In: $42^{\circ}$ Congresso da Sociedade Brasileira de Economia e Sociologia Rural, 2004, Cuiabá/MT. Anais..., Brasília/DF, v. 1, 2004.

CERQUEIRA, P. S.; ROCHA, A. G.; COELHO, V. P. Agricultura familiar e políticas públicas: algumas reflexões sobre o Programa de Aquisição de Alimentos no estado da Bahia. Revista Desenbahia, v. 3, n.5, p. 55-78, 2006.

COMPANHIA NACIONAL DE ABASTECIMENTO - CONAB. Gerência de Acompanhamento e Controle das Ações da Agricultura Familiar (GECAF). Total de produtos comercializados pelo PAA, nas modalidades CPRDoação, CPR-Estoque e CDAF, nos anos de 2009 a 2011. Brasília, 2012.

CONCEIÇÃO, J. C. P. R da. A influência de variáveis de mercado e de programas governamentais na determinação dos preços de produtos agrícolas. Texto para Discussão (IPEA), v. 1221, p. 7-13, 2006.

CONCEIÇÃO, J. C. P. R da. Contribuição dos novos instrumentos (contratos de Opção e PEP) para estabilização de preços e renda agrícola. Texto para Discussão (IPEA), v. 927, p. 1-23, 2002. 
CRUZ, A. C.; ALVARENGA, T. P. Avaliação da contribuição dos instrumentos COV e PEP para a estabilidade da renda agrícola. Revista de Estudos Sociais, v. 17, n. 33, 2015.

CRUZ, A. C.; TEIXEIRA, E. C. Desempenho da política de garantia de preços via contratos de opção de venda e prêmios para escoamento de produto. Revista de Política Agrícola, v. 15, p. 24-36, 2006.

CRUZ, A. C.; TEIXEIRA, E. C. Estabilização dos preços agrícolas: análise da eficácia dos instrumentos de apoio à comercialização agrícola. In: Anais $45^{\circ}$ Congresso da Sociedade Brasileira de Economia e Sociologia Rural, 2007, Londrina. Anais..., Brasília, 2007.

DIAS, T. F.; ROCHA, L. A. O Programa de Aquisição de Alimentos (PAA) da agricultura familiar e seus efeitos nos municípios do Rio Grande do Norte 2005 a 2011. Administração Pública e Gestão Social, v. 7, n.1, p. 16-25, 2015.

DORETTO, M.; MICHELLON, E. Avaliação dos Impactos Econômicos, Sociais e Culturais do Programa de Aquisição de Alimentos no Paraná. In: Flávio Borges Botelho Filho / Amauri Daros de Carvalho. (Org.). Avaliação de Políticas de Aquisição de Alimentos. 27 ed. Brasília: UnB / CEAM / NER, v. 7, p. 107-138, 2007.

GRAZIANO DA SILVA, J.; DEL GROSSI, M.E.; DE FRANÇA, C.G. Fome Zero: la experiencia brasileña. MDA: Brasília, 2012.

GRISA, C. As redes e as instituições do Programa de Aquisição de Alimentos. Revista Brasileira de Gestão e Desenvolvimento Regional, v. 6, n. 2, 2010.

IBGE - Instituto Brasileiro de Geografia e Estatística. Censo agropecuário 2006: resultados preliminares, IBGE, 2015.

IPC-IG. Structured Demand and Smallholder Farmers in Brazil: the Case of PAA and PNAE. International Policy Centre for Inclusive Growth (IPC - IG) United Nations Development Programme, Brasilia, 2013.

MACIEL, L. R. Mercado Institucional de Alimentos: Potencialidades e Limites para a Agricultura Familiar. Brasília: Faculdade de Agronomia e Medicina Veterinária, Universidade de Brasília, 2008, 115p. Dissertação de Mestrado.

MAGALHÃES, A. M.; SOARES, A. Os impactos do PAA-Leite sobre o Preço, a Produção e a renda da Pecuária Leiteira. Cadernos de Estudos Desenvolvimento Social em Debate, Brasília, n. 3, p. 2006.

MARTINS, S.P.; CAVALCANTI, L.I. Avaliação do impacto da execução do PAA no Estado do Rio Grande do Norte. Sociedade e Desenvolvimento Rural, v.1, n.1, 2007.

MATTEI, L. Programa de Aquisição de Alimentos da Agricultura Familiar (PAA): antecedentes, concepção e composição geral do Programa. Cadernos do CEAM (UnB), v. 7, p. 33-44, 2007 a. 
MATTEI, L. Políticas de apoio ao desenvolvimento da agricultura familiar no Brasil: o caso recente do Pronaf. Revista Econômica do Nordeste, Fortaleza, v. 38 , n. 1 , p. $143-158,2007$ b.

MDA. Ministério do Desenvolvimento Agrário. Brasília, 2015. Disponível em: <http://www.mda.gov.br/>. Acesso em: 16 de jul. 2015.

MEC. Ministério da Educação. Brasília, 2015. Disponível em: <http://portal.mec.gov.br/>. Acesso em: 16 de jul. 2015.

MILHORANCE, C.; GABAS, J. J. Reframing development from the South? A debate on the internationalization of Brazil's rural policies. In: International Conference of Public Policy, 2015, Milão. Anais..., Milão, 2015.

MÜLLER, A. L.; SILVA, M. K.; SCHNEIDER, S. A. A construção de políticas públicas para a agricultura familiar no Brasil: o Programa de Aquisição de Alimentos. Revista Estudos Sociedade e Agricultura, Rio de Janeiro, v. 20, 2012.

NUNES, S. P. O crédito rural do Pronaf e os recentes instrumentos de política agrícola para a agricultura familiar. Deser-Boletim Eletrônico, Curitiba, n. 156, p. 1-10, fev. 2007.

ORTEGA, A.C; JESUS, C.M.; SÓ, L.L.S. O PAA-leite na Bahia e em Minas Gerais: uma avaliação preliminar de seus modelos de implementação. Cadernos do CEAM. v. 5, n. 24, p. 57-89, ago. 2006.

PEREIRA, M. E. B. G.; LOURENZANI, A. E. B. S. Desafios e perspectivas do programa de aquisição de alimentos no município de Tupã - SP. Ciência e Natura, v. 36, n. 2, p. 230-240, mai./ago. 2014.

PIMENTEL-GOMES, F. Curso de estatística experimental. $15^{a}$ ed., São Paulo: FEALQ, 2009. 451 p.

SACCO DOS ANJOS, F.; BECKER, C. Agricultura familiar e mercados institucionais: o desenvolvimento como liberdade. Revista Econômica do Nordeste, v. 45, p. 92-101, 2014. Suplemento Especial.

SALGADO, R. J. S. F.; DIAS, M. M. Análise da influência do Programa de Aquisição de Alimentos (PAA) sobre a qualidade de vida de agricultores familiares do município de Viçosa/MG. Perspectivas em Políticas Públicas, Belo Horizonte, v. 6, n. 11, 2013.

SANTANA, A; C. Métodos Quantitativos em economia: elementos e aplicações. Belém: UFRA, 2003.

SCHMITT, C. J. Aquisição de alimentos da agricultura familiar: integração entre política agrícola e segurança alimentar e nutricional. Revista de Política Agrícola, v. 14, n.2, p. 78-88, abr./mai./jun. 2005.

SIMÃO, G. L.; SILVA, E. A.; SILVEIRA, S. F. R. Grau de cobertura do Programa de Aquisição de Alimentos (PAA) junto aos agricultores familiares do estado de Minas Gerais. Revista de Economia e Sociologia Rural, Piracicaba, v. 52, n. 03, p. 533-548, jul./set. 2014. 
SONNINO, R.; TORRES, C. L.; SCHNEIDER, S. Reflexive governance for food security: The example of school feeding in Brazil. Journal of Rural Studies, v. 36, p. 1-12, 2014.

SWENSSON, L. F. J. Institutional procurement of food from smallholder farmers: The case of Brazil. Food and Agriculture Organization of The United Nations, 2015.

YAZBEK, M. C. O Programa Fome Zero no Contexto das Políticas Sociais Brasileiras. São Paulo em Perspectiva, São Paulo, v. 18, n. 2, p. 104-112, jun. 2004. 